\title{
NEW MEASUREMENTS OF POSITIONS FOR THE ASTROGRAPHIC CATALOGUE STARS
}

\author{
G.A.IVANOV, V.S.KISLYUK, L.K.PAKULYAK, A.V.SERGEEV, \\ T.P.SERGEEVA and A.I.YATSENKO
}

Main Astronomical Observatory, National Academy of Sciences of Ukraine, Golosiiv, 252650 Kiev-22, Ukraine, maouas@gluk.apc.org

Six observatories of the former USSR carry out photographic observations within the FON project (the fourfold coverage of the northern sky) since 1982. The same type wide-angle Zeiss astrographs $(\mathrm{D}=400 \mathrm{~mm}, \mathrm{~F}=2000$ and 3000 $\mathrm{mm}$ ) are used. At present Golosiiv Observatory almost finished the Kiev observational part of the project and the measurements of onefold coverage plates have been started. The special measuring machine PARSEC (Programming Automatic Radial-Scanning Coordinatometer) has recently been constructed for this purpose.

We intend to apply the measurements of the FON project plates to the determination of new positions and proper motions of all stars in the Astrographic Catalogue (AC) using the last one as the first epoch of observations. AC is applied also as input catalogue for PARSEC. About 500 plates have been measured at the moment and proper motions for more than 500000 stars in different areas of the northern sky have been obtained.

The accuracy of the FON positions and proper motions of stars from the measurements of onefold coverage plates is estimated to be 0.35 arcsec and $0.006 \mathrm{arcsec} / \mathrm{yr}$, respectively.

Futher investigations will be carried out in cooperation with Sternberg Astronomical Institute (Moscow, Russia) and Astronomisches Rechen-Institut (Heidelberg, Germany).

The above-described reasearches became possible partly by Grant N V41000 from the International Science Foundation. 